\title{
Impact of Oscillator Noise in Bistatic and Multistatic SAR
}

\author{
Gerhard Krieger, Member, IEEE, and Marwan Younis, Member, IEEE
}

\begin{abstract}
This letter addresses the impact of limited oscillator stability in bistatic and multistatic synthetic aperture radars (SARs). Oscillator noise deserves special attention in distributed SAR systems since there is no cancellation of lowfrequency phase errors as in a monostatic SAR, where the same oscillator signal is used for modulation and demodulation. It is shown that the uncompensated phase noise may cause a timevariant shift, spurious sidelobes, and a broadening of the impulse response, as well as a low-frequency phase modulation of the focused SAR signal. Quantitative estimates are derived analytically for each of these errors based on a system-theoretic model taking into account the second-order statistics of the oscillator phase noise.
\end{abstract}

Index Terms-Bistatic radar, interferometry, multistatic radar, oscillator, phase noise, synchronization, synthetic aperture radar.

\section{INTRODUCTION}

B ISTATIC and multistatic synthetic aperture radar (SAR) systems operate with transmit and receive antennas that are mounted on different platforms [1], [2]. Such a spatial separation has several operational advantages that will increase the capability, reliability, and flexibility of future spaceborne SAR missions [3], [4]. Powerful applications of bistatic and multistatic satellite configurations include single-pass cross-track and along-track interferometry, high-resolution wide swath SAR imaging, bistatic imaging for improved scene classification, resolution enhancement, SAR tomography, and frequent monitoring [4]. However, the implementation of bistatic and multistatic SAR missions also raises a couple of new challenges like collision avoidance in close satellite formations, orbit design for the provision of appropriate baselines, increased susceptibility to ambiguities, and instrument synchronization [4]-[12].

This letter addresses the impact of limited oscillator stability during bistatic and multistatic SAR data acquisitions. Oscillator errors deserve special attention in distributed SAR systems since there is no cancellation of low-frequency phase errors as in a monostatic SAR, where the same oscillator signal is used for modulation and demodulation [7]. For a quantitative investigation, we introduce in Section II a system-theoretic model that describes residual phase errors of ultrastable oscillators (USOs) in the framework of stochastic processes. In Section III, it is then shown that uncompensated phase noise may cause a timevariant shift, spurious sidelobes, and a broadening of the bistatic impulse response, as well as a low-frequency phase modulation of the focused SAR signal. The error for each contribution is derived analytically by an appropriately weighed integration of

Manuscript received November 22, 2005; revised January 9, 2006.

The authors are with the German Aerospace Center, Microwaves and Radar Institute, 82234 Oberpfaffenhofen, Germany (e-mail: Gerhard.Krieger@ dlr.de).

Digital Object Identifier 10.1109/LGRS.2006.874164

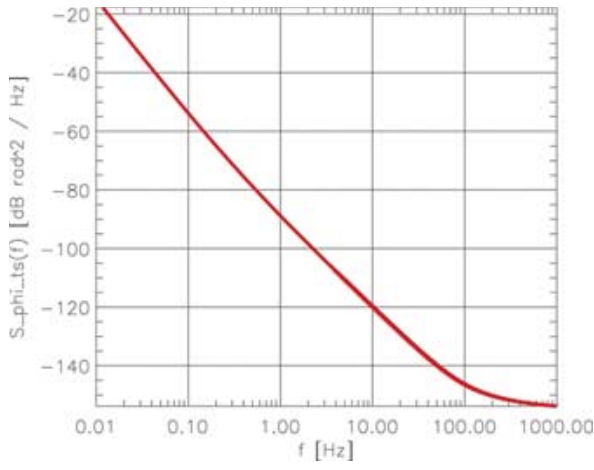

Fig. 1. Power spectral density $S_{\varphi}^{\mathrm{TS}}(f)$ of oscillator phase noise. Lowfrequency values correspond to an Allan standard deviation [13] with $\sigma_{a}(\tau=$ $1 \mathrm{~s}) \approx 1 \times 10^{-11}, \sigma_{a}(\tau=10 \mathrm{~s}) \approx 2 \times 10^{-11}$, and $\sigma_{a}(\tau=100 \mathrm{~s}) \approx$ $6 \times 10^{-11}$, and higher frequency values correspond to single sideband to carrier phase noise ratios of $L(f=1 \mathrm{~Hz}) \approx-90 \mathrm{dBc} / \mathrm{Hz}$ and $L(f=10 \mathrm{~Hz}) \approx$ $-120 \mathrm{dBc} / \mathrm{Hz}$

the power spectral density that models the second-order phase fluctuations of the independent USOs. The letter is concluded in Section IV with a discussion of potential synchronization strategies for bistatic and multistatic SAR systems.

\section{Modeling Oscillator Phase ERrors}

Random phase noise is often modeled by a second-order stationary stochastic process, which is conveniently characterized in the Fourier frequency domain by its power spectral density $S_{\varphi}^{\mathrm{TS}}(f)$, where $S_{\varphi}^{\mathrm{TS}}(f)$ describes the two-sided spectral density of phase fluctuations in radians squared per hertz bandwidth at Fourier frequency $f$ from the carrier [13]-[16]. Fig. 1 shows as an example a typical phase spectrum $S_{\varphi}^{\mathrm{TS}}(f)$ of a USO with a frequency of $f_{\text {osc }}=10 \mathrm{MHz}$.

The phase spectrum in Fig. 1 can analytically be expressed by a composite power law model [13]-[16]

$$
S_{\varphi}^{\mathrm{TS}}(f)=a f^{-4}+b f^{-3}+c f^{-2}+d f^{-1}+e
$$

where the coefficients $a$ to $e$ describe contributions from: 1) random walk frequency noise; 2) frequency flicker noise; 3) white frequency noise; 4) flicker phase noise; and 5) white phase noise, respectively. ${ }^{1}$ Instead of employing $S_{\varphi}^{\mathrm{TS}}(f)$, it is often more convenient to use the one-sided power spectral density $S_{\varphi}(f)$, which is related to $S_{\varphi}^{\mathrm{TS}}(f)$ by $S_{\varphi}(f)=2 S_{\varphi}^{\mathrm{TS}}(f)$ for $f>0$ and $S_{\varphi}(f)=0$ for $f<0$. Fig. 2 shows simulation examples of the predicted oscillator phase errors for a time interval of $1 \mathrm{~min}$. Note that the contribution from a linear phase

\footnotetext{
${ }^{1} S_{\varphi}^{\mathrm{TS}}(f)$ in Fig. 1 uses $\{a=-95 \mathrm{~dB}, b=-90 \mathrm{~dB}, c=-200 \mathrm{~dB}, d=$ $-130 \mathrm{~dB}, e=-155 \mathrm{~dB}\}$, which can be regarded as a representative example for the USO of current spaceborne SAR systems.
} 

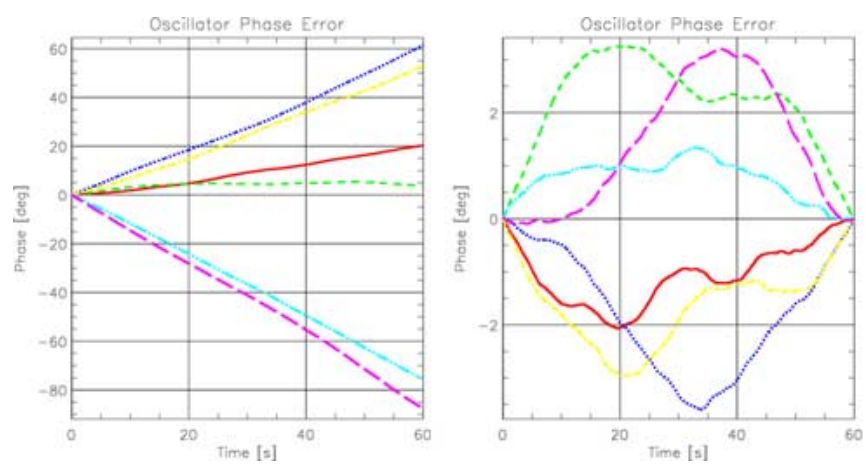

Fig. 2. Example of simulated oscillator phase errors. Shown are six 1-min excerpts of a 90-min realization of the stochastic process defined in Fig. 1 (left) before and (right) after subtraction of linear phase ramps.
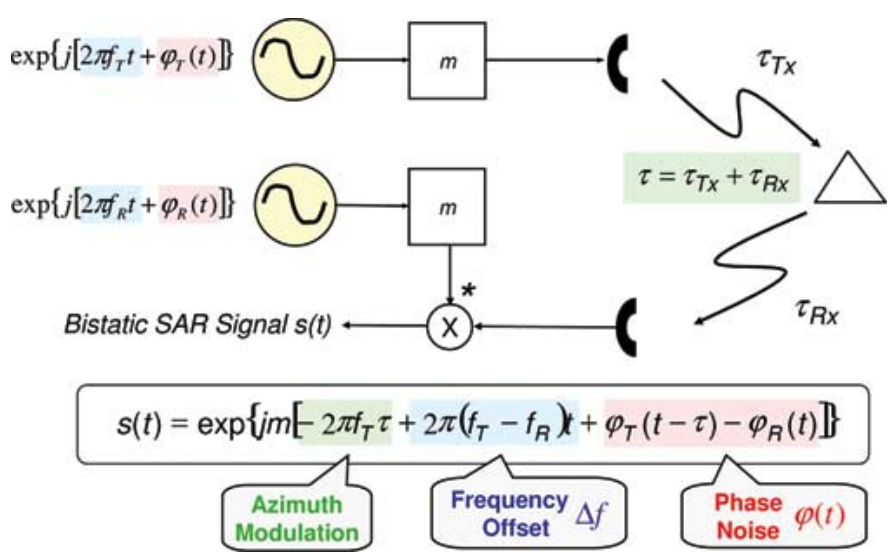

Fig. 3. Derivation of baseband bistatic phase errors after demodulation. ${ }^{2} f_{T}$ and $f_{R}$ denote the transmit and receive oscillator frequencies, $\varphi_{T}$ and $\varphi_{R}$ are the corresponding phase errors, and $\tau$ is the traveling time of the radar pulse.

ramp corresponding to a frequency offset has been suppressed in the right image for better illustration.

Estimates of the bistatic phase errors in the RF band can be obtained by multiplying the realizations in Fig. 2 by $\sqrt{ } 2 m$, where $m=f_{0} / f_{\text {osc }}$ is the frequency up-conversion factor and $f_{0}$ is the radar carrier frequency (cf. illustration in Fig. 3). This phase scaling corresponds to a multiplication of the phase power spectral density $S_{\varphi}(f)$ by $2 m^{2}$, where we implicitly assume uncorrelated oscillators with equal $S_{\varphi}(f)$ (cf. [15]).

\section{IMPACT OF PhASE ERRORS IN BISTATIC SAR}

After bistatic SAR processing, oscillator phase errors manifest themselves as a deterioration of the impulse response function (IRF). Note that this deterioration will mainly impact the azimuth response due to the relatively higher quality of oscillator performance over shorter time scales. Typical disturbances are a time-variant shift of the main lobe, spurious sidelobes, and a broadening of the impulse response, as well as phase errors in the focused SAR signal [17]-[19]. Fig. 4 illustrates the impact of oscillator noise on azimuth focusing for a coherent integration time of $T_{a}=2 \mathrm{~s}$ assuming an X-band

${ }^{2}$ This derivation assumes ideal frequency up- and down-conversion. A more thorough analysis could incorporate into $S_{\varphi}(f)$, e.g., the specification of a potential phase-locked oscillator (PLO) used for frequency conversion. However, for the coherent integration times considered in this letter, the phase noise from the USO will be dominant due to the limited PLO loop bandwidth.

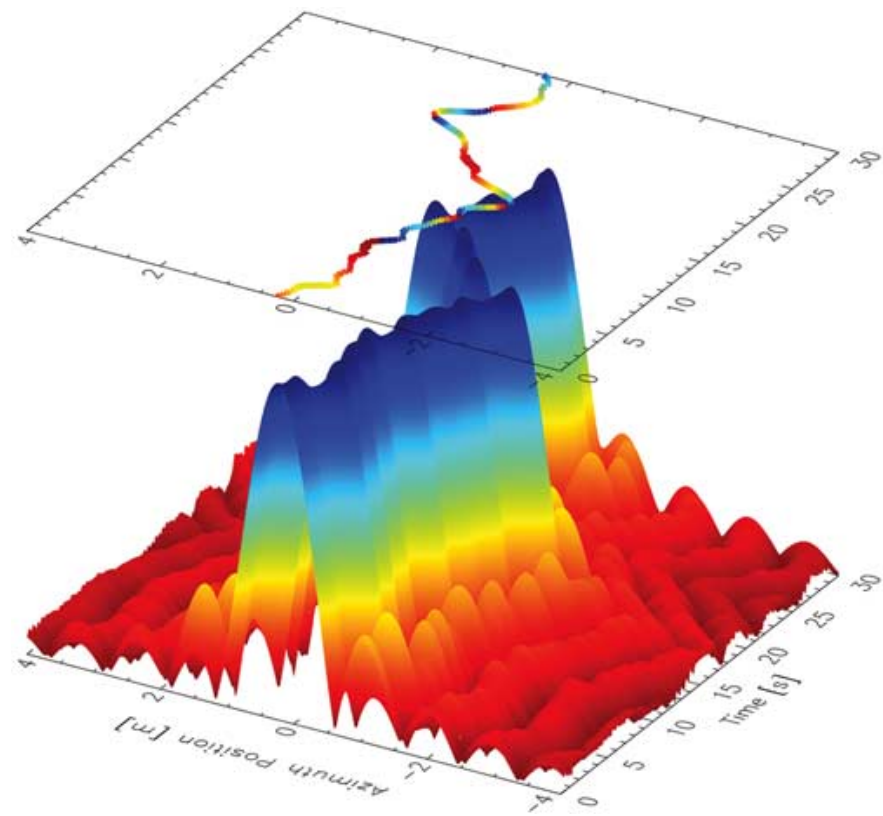

Fig. 4. Temporal evolution of the focused azimuth response of a fictious bistatic SAR with a 2-s integration time $\left(v_{\mathrm{sat}}=7 \mathrm{~km} / \mathrm{s}, r_{0}=800 \mathrm{~km}, \lambda=\right.$ $3.1 \mathrm{~cm}$ ). In this simulation, the noise model in Fig. 1 has been used. The colored curve on the top shows the temporal variation of the main lobe phase.

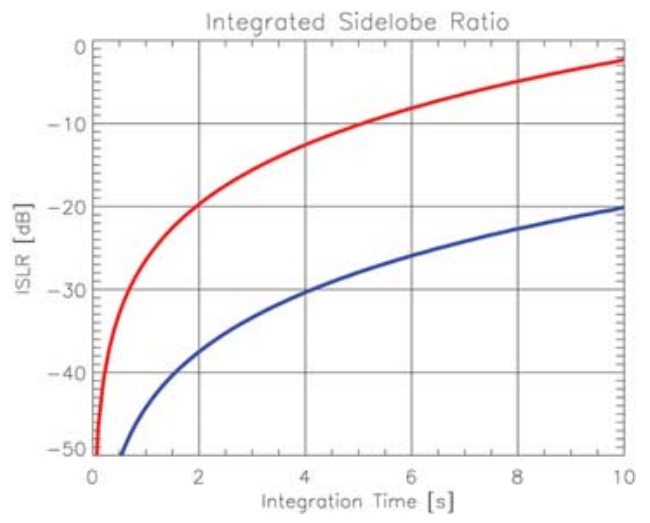

Fig. 5. ISLR for (red) X-band and (blue) L-band.

sensor (no weighing has been used). It becomes evident that the oscillator phase noise may not only defocus the SAR image in the azimuth but also introduce significant positioning and phase errors along the scene extension. The following subsections derive analytic expressions for each of these errors.

\section{A. Spurious Sidelobes}

High-frequency phase noise will cause spurious sidelobes in the IRF. This deterioration can be characterized by an increase of the integrated sidelobe ratio (ISLR), which measures the energy in the impulse response sidelobes relative to the energy in the main lobe [20]. For an azimuth integration time $T_{a}$, the deterioration of the ISLR may be approximated from the phase spectrum as [1], [7], [17], [18]

$$
\operatorname{ISLR} \approx \sigma_{\varphi, \mathrm{HF}}^{2}\left(f>\frac{1}{T_{a}}\right)=2\left(\frac{f_{0}}{f_{\mathrm{osc}}}\right)^{2} \int_{1 / T_{a}}^{\infty} S_{\varphi}(f) d f .
$$




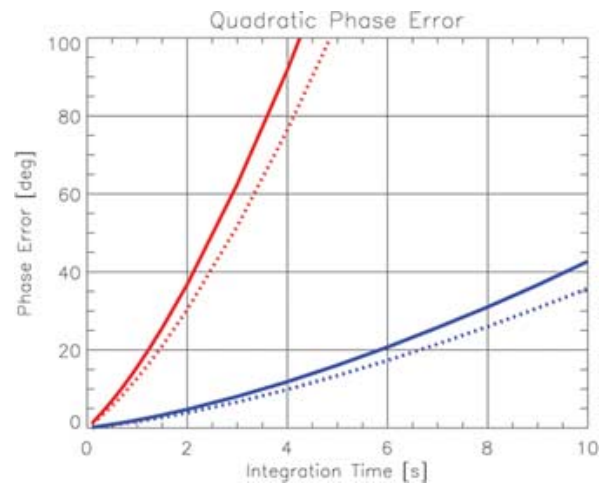

Fig. 6. Quadratic phase errors for (red) X-band and (blue) L-band. Solid lines are for (3), and dotted lines are for the formula in footnote 3 .

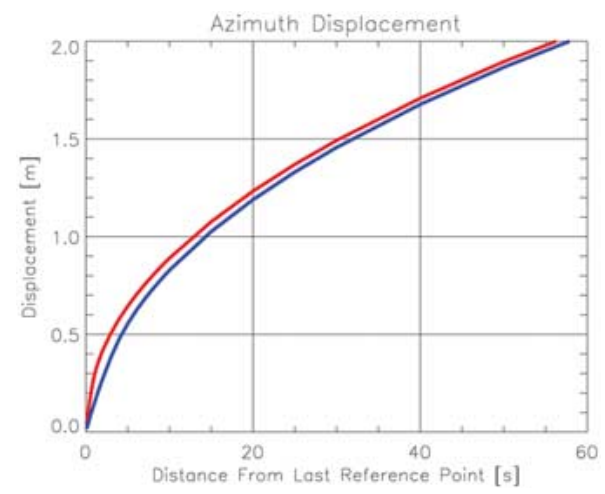

Fig. 7. Azimuth displacement as a function of the distance from the last reference point for (red) X-band and (blue) L-band. The coherent integration times are $T_{a}=1 \mathrm{~s}$ for the X-band and $T_{a}=4 \mathrm{~s}$ for the L-band $\left(r_{0}=800 \mathrm{~km}\right.$ and $v=7 \mathrm{~km} / \mathrm{s}$ ).

The factor 2 is due to the use of two independent oscillators, and the scaling factor in the parentheses is due to the multiplication of the oscillator frequency $f_{\text {osc }}$ with $\left(f_{0} / f_{\text {osc }}\right)$ to obtain the radar signal with center (carrier) frequency $f_{0}$. The upper integration limit may be substituted by the inverse of the transmit pulse duration since higher frequency phase errors are averaged during range compression. Fig. 5 shows estimates of the ISLR for the phase spectrum given in Fig. 1. A typical requirement for the maximum tolerable ISLR is $-25 \mathrm{~dB}$, which would enable a maximum coherent integration time $T_{a}$ of approximately $1 \mathrm{~s}$ in the $\mathrm{X}$-band and $6 \mathrm{~s}$ in the L-band.

\section{B. Main Lobe Dispersion}

Quadratic phase errors cause a broadening of the azimuth response. An approximation of these errors may be derived by expanding the phase for each frequency component of the stochastic process in a second-order Taylor series [17]-[19] $]^{3}$

$$
\sigma_{Q}^{2}=2\left(\frac{f_{0}}{f_{\text {osc }}}\right)^{2} \frac{\left(\pi T_{a}\right)^{4}}{4} \int_{0}^{1 / T_{a}} f^{4} S_{\varphi}(f) d f .
$$

\footnotetext{
${ }^{3}$ This is the adapted formula from [17], which overestimates the contributions for $f>0.2 / T_{a}$. A more accurate prediction can analytically be derived from a second-order least squares polynomial fit that approximates the realizations $\varphi(t)$ by $p(t)=a t^{2}+b t+c$ within the time interval $\left[t-T_{a} / 2, t+\right.$ $\left.T_{a} / 2\right]$, i.e., $\quad \sigma_{Q}^{2}=\left(f_{0} / f_{\text {osc }}\right)^{2} \int_{0}^{\infty} S_{\varphi}(f)\left(225 / 2\left(\pi f T_{a}\right)^{6}\right)\left[3 \pi f T_{a} \times\right.$ $\left.\cos \left(\pi f T_{a}\right)+\left(\left(\pi f T_{a}\right)^{2}-3\right) \sin \left(\pi f T_{a}\right)\right]^{2} d f$.
}
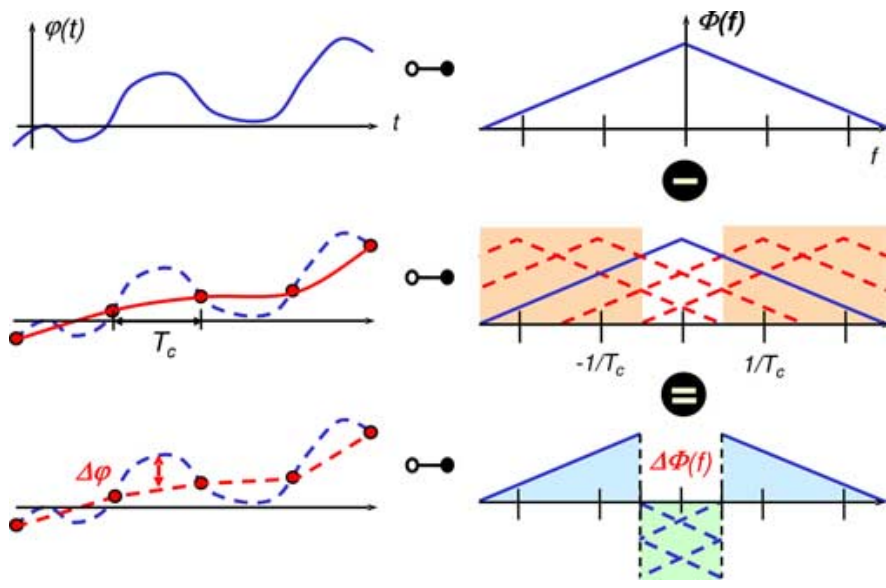

Fig. 8. Illustration of the major steps in the derivation of residual phase errors after periodic phase synchronization. The left column shows operations in the time domain, and the right column illustrates the corresponding operations in the frequency domain.

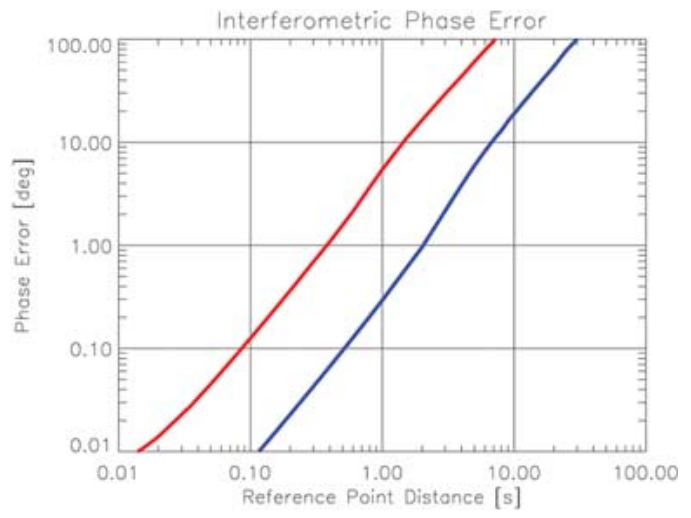

Fig. 9. Bistatic phase error for (red) X-band and (blue) L-band after periodic phase synchronization prior to bistatic SAR focusing.

A typical requirement for quadratic phase errors is $\sigma_{Q}<$ $\pi / 4$, which leads to a resolution loss of $3 \%$ in case of unweighed azimuth processing. ${ }^{4}$ Fig. 6 shows estimates of the quadratic phase errors in the X-band and L-band for the phase spectrum $S_{\varphi}(f)$ in Fig. 1. In this example, a coherent integration time up to $2.5 \mathrm{~s}$ would still be tolerable in the X-band, ensuring good bistatic focusing of the impulse response.

\section{Azimuth Displacement}

Any difference between the oscillator frequencies will cause a shift of the bistatic impulse response in azimuth. Assuming a nonsquinted quasi-monostatic imaging geometry, the azimuth shift is given by

$$
\Delta x=\frac{c_{0} r_{0}}{2 v} \frac{\Delta f}{f_{\mathrm{osc}}}
$$

where $v$ is the platform velocity, $r_{0}$ is the slant range, and $\left(\Delta f / f_{\text {osc }}\right)$ is the relative frequency deviation between the two USOs. Note that a frequency deviation of only $1 \mathrm{~Hz}$ between two $10-\mathrm{MHz}$ oscillators (corresponding to a relative frequency

\footnotetext{
${ }^{4}$ The azimuth dispersion can be approximated by ( $\Delta a z_{\text {bistat }} /$ $\left.\Delta a z_{\text {ideal }}\right)^{2}=1+\left(\sigma_{Q} / \pi\right)^{2}$. Quadratic phase errors will furthermore introduce phase errors in the focused SAR response, which are one third of $\sigma_{Q}$ [21].
} 


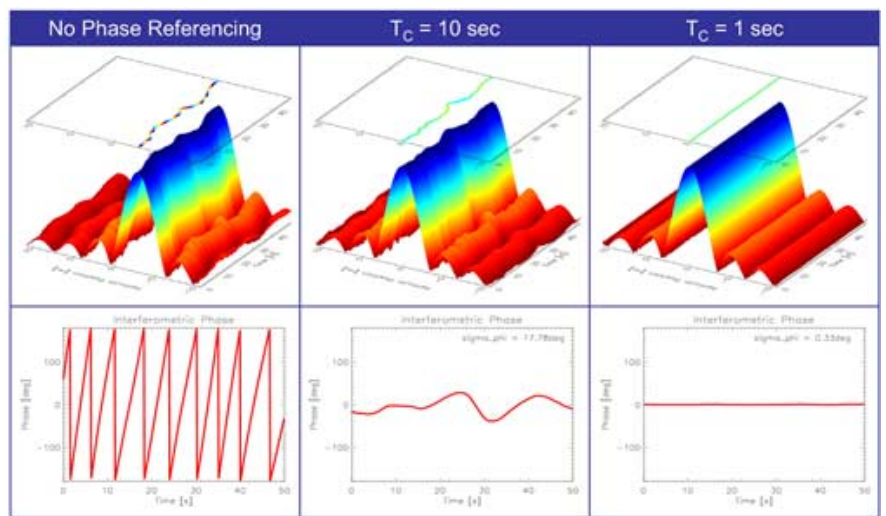

Fig. 10. Simulated bistatic SAR impulse response in the L-band. The upper plots show the simulated profile of the azimuth response (left) without synchronization, (middle) a phase referencing every $10 \mathrm{~s}$, and (right) a phase referencing every second. The lower plots show the corresponding impulse response phase errors. The root mean square phase errors are $17.78^{\circ}$ for $T_{C}=10 \mathrm{~s}$ and $0.33^{\circ}$ for $T_{C}=1 \mathrm{~s}$.

deviation of $10^{-7}$ ) causes a constant azimuth shift of $\Delta x \approx$ $1.7 \mathrm{~km}$ for $v=7 \mathrm{~km} / \mathrm{s}$ and $r_{0}=800 \mathrm{~km}$. A coarse estimate of $\Delta f$ can be obtained from a spectral analysis of the demodulated azimuth signal (assuming a known Doppler centroid). A precise measurement of the azimuth displacement requires ground control points or an appropriate phase referencing system. A further opportunity arises in systems with simultaneous monostatic and bistatic SAR data acquisition (as, e.g., TanDEM-X [22]), where it is possible to estimate $\Delta f$ via a coregistration between monostatic and bistatic SAR images. The variance of the remaining azimuth shift may then be derived from the spectral representation of the Allan variance with nonadjacent samples [14] as

$$
\begin{aligned}
\sigma_{\Delta x}^{2}(t)=2 & \left(\frac{c_{0} r_{0}}{v}\right)^{2} \int_{0}^{\infty} \frac{f^{2}}{f_{\mathrm{osc}}^{2}} S_{\varphi}(f) \\
& \times\left(\frac{\sin \left(\pi T_{a} f\right)}{\pi T_{a} f}\right)^{2}\left[1-\left(\frac{\sin (2 \pi f t)}{2 \sin (\pi f t)}\right)^{2}\right] d f
\end{aligned}
$$

where $t$ is the time interval elapsed from the last reference point. As explained in [13], the $\operatorname{sinc}\left(\pi T_{a} f\right)$ function is due to averaging over $T_{a}$ and the $\sin (2 \pi f t) / \sin (\pi f t)$ function is due to the time delay $t$ between the two "frequency" measurements. Fig. 7 shows the standard deviation of the predicted azimuth shift for the phase spectrum in Fig. 1 as a function of $t$. Note that the azimuth shift is independent of the wavelength but depends slightly on the coherent integration time.

\section{Range Displacement}

The range shift will be dominated by deviations between the pulse repetition frequencies (PRFs) of the transmitter and receiver. Since the PRF is usually derived from the local oscillator by appropriate frequency division, the shift in slant range may be expressed as $\Delta r(t)=c_{0} \Delta f t / 2 f_{\text {osc }}$, where we assume again a quasi monostatic imaging geometry. A frequency deviation of $\Delta f=1 \mathrm{~Hz}$ between the two 10-MHz oscillators will hence cause a linear range drift of $15 \mathrm{~m} / \mathrm{s}$. From this, it becomes clear that already small frequency deviations between the two oscillators may cause rather large range shifts for long data acquisitions. As a first consequence, this will require an adaptation of the recording window to the transmit event, e.g., by periodic PRF synchronization using either a communication link [7] or GPS signals [5], or, as an alternative, continuous recording. Precise range measurements will furthermore require an accurate time referencing between the transmitter and receiver. Possible solutions for precise time synchronization on a nanosecond level are discussed in [1], [8], [11], and [12]. An alternative is the recourse to calibration targets on the ground. The residual range shift $\Delta r$ between such reference points can then be estimated from $\Delta r=(\lambda / 4 \pi) \Delta \varphi$, where $\Delta \varphi$ corresponds to the residual bistatic phase error between the references. An estimate of $\Delta \varphi$ as a function of the distance between calibration targets will be provided in the next section.

\section{E. Phase Errors of the Bistatic Impulse Response}

For the estimation of the bistatic phase error $\Delta \varphi$, we assume the availability of a regular grid of phase reference values separated by a (temporal) distance $T_{c}$. Such references can, e.g., be obtained by a pulsed synchronization link (cf. TanDEM-X [22]) or in an interferometric system via the availability of appropriate ground control points. The phase samples will then allow for the correction of low-frequency bistatic phase errors via $\sin (x) / x$ interpolation. ${ }^{5}$ The variance of the residual bistatic phase errors between the phase references can be derived as follows.

We start with an analysis of the periodic phase synchronization prior to bistatic SAR processing. The typical RF phase error of the bistatic raw data is then given by a realization $\varphi(t)$ of the stochastic process $\left\{2\left(f_{0} / f_{\text {osc }}\right)^{2} S_{\varphi}(f)\right\}$. As shown in the first row of Fig. 8, the realization $\varphi(t)$ has in the frequency domain the corresponding spectrum $\Phi(f)$. As a next step, we assume the availability of phase references separated by $T_{c}$. These reference points can be regarded as samples of the bistatic RF phase error function $\varphi(t)$. In the frequency domain, periodic sampling causes a repetition of the corresponding phase error spectrum $\Phi(f)$. The samples are then interpolated by a $\sin (x) / x$ function that corresponds to an ideal low-pass filter with a cutoff frequency $1 /\left(2 T_{c}\right)$. As illustrated in Fig. 8 on the right, the remaining phase errors after synchronization can finally be computed in the frequency domain by subtracting the repeated and low-pass-filtered spectrum of the interpolated signal from the original spectrum $\Phi(f)$ as

$$
\Delta \Phi(f)=\left\{\begin{array}{cl}
\Phi(f) & \text { for }|f|>1 /\left(2 T_{c}\right) \\
\sum_{i=1}^{\infty} \Phi\left(f+i / T_{c}\right)+\Phi\left(f-i / T_{c}\right) & \text { for }|f| \leq 1 /\left(2 T_{c}\right)
\end{array}\right.
$$

The phase errors after bistatic SAR processing can be estimated with sufficient accuracy by integrating the phase errors over a time interval $T_{a}{ }^{6}{ }^{6}$ This integration corresponds to a low-pass filter with a $\sin \left(\pi T_{a} f\right) /\left(\pi T_{a} f\right)$ transfer function. The

\footnotetext{
${ }^{5}$ The phase errors after linear interpolation between two reference points can be obtained by computing the interpolation error for each sinusoidal component individually. The variance of the total error is then given by

$$
\sigma_{\Delta \varphi}^{2}=2\left(\frac{f_{0}}{f_{\text {osc }}}\right)^{2} \int_{0}^{\infty} S_{\varphi}(f)\left[2-\frac{6+2 f^{2} \pi^{2} T_{c}^{2}}{3 f^{2} \pi^{2} T_{c}^{2}} \sin ^{2}\left(\pi f T_{c}\right)\right] d f .
$$

${ }^{6}$ This approximate computation of the output phase is valid for small residual
} phase error variations where $\exp (j \varphi(t)) \approx 1+j \varphi(t)$. 
variance of the phase errors is then given by ${ }^{7}$

$$
\begin{aligned}
\sigma_{\Delta \varphi}^{2}= & 2\left(\frac{f_{0}}{f_{\mathrm{osc}}}\right)^{2}\left[\int_{1 /\left(2 T_{c}\right)}^{\infty} S_{\varphi}(f)\left(\frac{\sin \left(\pi T_{a} f\right)}{\pi T_{a} f}\right)^{2} d f\right. \\
& \left.+\sum_{i=1}^{\infty} \int_{-1 /\left(2 T_{c}\right)}^{1 /\left(2 T_{c}\right)} S_{\varphi}\left(f+\frac{i}{T_{c}}\right)\left(\frac{\sin \left(\pi T_{a} f\right)}{\pi T_{a} f}\right)^{2} d f\right]
\end{aligned}
$$

where the first integral describes the high-frequency phase errors (contribution from the blue triangles in the lower right of Fig. 8) and the second integral corresponds to the lowfrequency phase errors caused by aliasing artifacts due the periodic repetition of the spectrum $\Phi(f)$ (contribution from the green square in the lower right spectrum of Fig. 8). In case of a posteriori phase correction, i.e., a phase correction after bistatic SAR focusing, the residual phase errors can be estimated by substituting $f$ with $f+i / T_{c}$ in the $\sin \left(\pi T_{a} f\right) /\left(\pi T_{a} f\right)$ function on the second line of (7).

Fig. 9 shows the standard deviation of the predicted residual phase error for the spectrum $S_{\varphi}(f)$ in Fig. 1 as a function of $T_{c}$. It becomes clear that this error will rapidly increase with increasing reference point separation $T_{c}$. For example, a maximum tolerable phase error of $10^{\circ}$ would require a synchronization frequency of ca. $0.7 \mathrm{~Hz}$ in the X-band and $0.15 \mathrm{~Hz}$ in the L-band. Assuming a radar beam velocity of $7 \mathrm{~km} / \mathrm{s}$, this corresponds to a ground control point every $10 \mathrm{~km}$ in the X-band and every $50 \mathrm{~km}$ in the L-band. The phase errors in Fig. 9 can also be regarded as estimates of the interferometric phase errors arising in both TanDEM-like and Cartwheel-like satellite configurations, where the former combine one "errorfree" monostatic image with one "erroneous" bistatic image, and the latter use one common transmitter in combination with two independent receivers.

Fig. 10 shows simulations of the bistatic impulse response without phase referencing (left) and with synchronization for $T_{c}=10 \mathrm{~s}$ (middle) and $T_{c}=1 \mathrm{~s}$ (right). Note that phase errors of $17.78^{\circ}$ and $0.33^{\circ}$ fit quite well with the analytic predictions in Fig. 9, which have been obtained from (7).

\section{Discussion}

This letter analyzed the impact of oscillator phase noise in bistatic and multistatic SARs. Based on a second-order stochastic model, quantitative estimates have been derived for potential errors like a distortion of the bistatic SAR impulse response, azimuth and range displacements, as well as interferometric phase errors. The most demanding requirements arise from multistatic interferometry. For example, the generation of high-resolution digital elevation models (DEMs) will require precise relative phase knowledge in the order of a few degrees to avoid a low-frequency modulation of the DEM in azimuth [22]. Such errors require an appropriate phase referencing between the independent USOs. As can be seen from Fig. 9, the required update frequency of an $\mathrm{X}$-band interferometer is in the order of $1-10 \mathrm{~Hz}$ for the oscillator characterized in Fig. 1. This requirement is reduced for longer wavelengths.

\footnotetext{
${ }^{7}$ The integration and summation limits in (7) take into account that $S_{\varphi}(f)$ has been defined as the one-sided spectral density of the phase fluctuations.
}

Possible solutions for phase referencing in an interferometric SAR are a direct exchange of radar pulses or a ping-pong interferometric mode in case of fully active systems, and an appropriate bidirectional phase synchronization link in case of semi-active constellations. Various implementations of a bidirectional synchronization link are analyzed in detail in a following letter [23], taking into account additional phase errors from the synchronization hardware. An alternative to such relative phase referencing solutions is the use of oscillators with a significantly improved long-term frequency stability in combination with a sparse net of ground control points. For example, the space-qualified 5-MHz oscillators in [24] have a typical short-term stability of $\sigma_{a}(\tau=10 \mathrm{~s})=10^{-13}$, which would decrease the interferometric phase errors in Fig. 9 by two orders of magnitude.

\section{REFERENCES}

[1] N. Willis, Bistatic Radar. Boston, MA: Artech House, 1991.

[2] P. Hartl and H. M. Braun, Bistatic Radar in Space, in Space Based Radar Handbook, L. Cantafio, Ed. Norwood, MA: Artech House, 1989.

[3] M. Martin, P. Klupar, S. Kilberg, and J. Winter, "Techsat 21 and revolutionizing space missions using microsatellites," in Proc. 15th Amer. Inst. Aeronaut. Astronaut. Conf. Small Satell., Logan, UT, 2001, pp. 1-10.

[4] G. Krieger and A. Moreira, "Spaceborne bi- and multistatic SAR: Potentials and challenges," Proc. Inst. Elect. Eng., Radar Sonar Navigat., 2006, to be published.

[5] P. Dubois-Fernandez, H. Cantalloube, B. Vaizan, G. Krieger, R. Horn, M. Wendler, and V. Giroux, "ONERA-DLR bistatic SAR campaign: Planning, data acquisition, and first analysis of bistatic scattering behavior of natural and urban targets," Proc. Inst. Elect. Eng., Radar Sonar Navigat., 2006, to be published.

[6] D. Martinsek and R. Goldstein, "Bistatic radar experiment," in Proc. EUSAR, Friedrichshafen, Germany, 1998, pp. 31-33.

[7] J. L. Auterman, "Phase stability requirements for a bistatic SAR," in Proc. IEEE Nat. Radar Conf., Atlanta, GA, 1984, pp. 48-52.

[8] W. Lewandowski, J. Azoubib, and W. Klepzynski, "GPS: Primary tool for time transfer," Proc. IEEE, vol. 87, no. 1, pp. 163-172, Jan. 1999.

[9] M. Eineder, "Oscillator clock drift compensation in bistatic interferometric SAR," in Proc. IGARSS, Toulouse, France, 2003, pp. 1449-1451.

[10] J. Ender, I. Walterscheid, and A. Brenner, "New aspects of bistatic SAR: Processing and experiments," in Proc. IGARSS, Anchorage, AK, 2004, pp. 1758-1762.

[11] M. Weiß, "Time and frequency synchronisation aspects for bistatic SAR systems," in Proc. EUSAR, 2004, pp. 395-398.

[12] G. Yates, A. Horne, A. Blake, R. Middleton, and D. Andre, "Bistatic SAR image formation," in Proc. EUSAR, Ulm, Germany, 2004, pp. 581-584.

[13] J. Rutman, "Characterization of phase and frequency instabilities in precision frequency sources: Fifteen years of progress," Proc. IEEE, vol. 66, no. 9, pp. 1048-1073, Sep. 1978.

[14] J. Barnes, A. Chi, L. Cutler, et al., "Characterization of frequency stability," IEEE Trans. Instrum. Meas., vol. 20, no. 2, pp. 105-120, May 1971.

[15] F. Walls and D. Allan, "Measurements of frequency stability," Proc. IEEE, vol. 74 , no. 1 , pp. 162-168, Jan. 1986

[16] "Special issue on time and frequency," Proc. IEEE, vol. 79, Jul. 1991

[17] S. Buckreuss, "Bewegungskompensation für flugzeuggetragene SAR Systeme," DLR, Oberpfaffenhofen, Germany, DLR Res. Rep. 94-17, 1994.

[18] - "Motion errors in an airborne synthetic aperture radar system," ETT-J., vol. 2, no. 6, pp. 655-664, 1991.

[19] D. Hounam, E. Panula-Otto, and K. Wägel, "Oscillator Noise and SAR Image Quality," in "DLR Res. Report," 1994.

[20] W. Carrara, R. Goodman, and R. Majewski, Spotlight Synthetic Aperture Radar: Signal Processing Algorithms. Boston, MA: Artech House, 1995.

[21] I. Cumming and F. Wong, Digital Processing of Synthetic Aperture Radar Data. Boston, MA: Artech House, 2005.

[22] G. Krieger, H. Fiedler, I. Hajnsek, M. Eineder, M. Werner, and A. Moreira, "TanDEM-X: Mission concept and performance analysis," in Proc. IGARSS, 2005, pp. 4890-4893.

[23] M. Younis, R. Metzig, and G. Krieger, "Performance prediction of a phase synchronisation link for bistatic SAR," IEEE Geosci. Remote Sens. Lett., vol. 3, no. 3, Jul. 2006.

[24] V. Candelier, P. Canzian, J. Lamboley, M. Brunet, and G. Santarelli, "Space qualified $5 \mathrm{MHz}$ ultra stable oscillators," in Proc. Int. Freq. Control Symp., Tampa, FL, 2003, pp. 575-582. 